\title{
A comparison of modelling approaches for closed-loop decision making over discrete domains in manufacturing systems
}

\author{
Jenny L. Diaz C., Sorin Olaru, Senior Member IEEE, and Carlos Ocampo-Martinez, Senior Member IEEE
}

\begin{abstract}
The manufacturing industry is transforming towards smart, flexible, and energy-efficient systems. In this regard, control strategies based on optimisation have been proposed to improve the energy efficiency of manufacturing systems. Usually, the optimisation problem behind the controller design involves decision variables constrained to binary and discrete domains resulting in Mixed-Integer Linear Programming (MILP) problems and require a high computational burden to find their optimal solution. In this paper, three different approaches are proposed for the closed-loop decision making over discrete domains in manufacturing systems, including the constraints and limitations to their implementation. Thus, the number and nature of required variables as well as the additional constraints needed to represent the discrete domains of the decision variables are established. These approaches are tested and compared to solve the optimisation problem behind a predictive-like controller designed to minimise the energy consumption of a manufacturing process line.
\end{abstract}

\section{INTRODUCTION}

In many industrial processes, both the optimal design and the implementation of optimisation-based control techniques often require variables constrained to a discrete set of admissible values. Some relevant applications of the use of discrete input signals can be found in chemical processes [1], process planning and scheduling [2], smart grids [3], and on-off systems [4]. For the particular case of manufacturing systems, system actuators can be of on-off nature of having different activation levels or status [5]. Thus, both the problem formulation and discrete optimisation method are crucial to solve these optimisation problems in a reliable and fast way. This last fact is quite important in control applications subject to real-time implementation constraints [6].

The use of discrete decision variables would imply a high computational burden for the optimisation routines. In most of the control applications, decisions involving discrete actuators are removed from the control layer and dealt with logic rules, precomputed control laws, or heuristic approximations [7], [8], [9]. However, most of these approaches are not able to respond effectively to disturbances or changes in the system since they are predefined considering specific operational conditions. For the case of flexible manufacturing systems, in which their operational conditions can change, these

Jenny L. Diaz C. and Carlos Ocampo-Martinez are with Automatic Control Department, Universitat Politècnica de Catalunya, Institut de Robòtica i Informàtica Industrial (CSIC-UPC), Llorens i Artigas 4-6, 08028 Barcelona, Spain. (e-mail: \{jenny.lorena.diaz, carlos. ocampo\} @upc.edu)

Sorin Olaru is with the Laboratory of Signals and Systems (L2S, UMR CNRS 8506), Centrale Supélec-CNRS Université Paris Sud, Université Paris-Saclay, 91190 Gif-sur-Yvette, France. (e-mail: sorin.olaru@centralesupelec.fr) approaches could be not suitable. Thus, with advances in computer performance and optimisation software, the common way to solve such problems is by transforming them into Mixed-Integer Programming (MIP) problems and solving them by using branch-and-bound-based MILP solvers such as those available in packages like cplex and gurobi. Based on this approach, to choose among $n$ discrete values in an MIP problem, $n$ binary variables that sum up to one are required [10]. Nonetheless, if the optimisation problem is not properly formulated and the decision variables are not restricted effectively to the feasible alternative, this approach could lead to unbalanced branch-and-bound trees and may result in long solution times [11].

Different approaches have been proposed in the literature to reduce the number of decision variables and the computational time to solve the related problem [12]. However, in more complex cases, these approaches imply the product of some polynomial terms, thus losing the linearity. In [13], a set of equations for linearising discrete cross-product terms that can be incorporated into conventional MILP reformulations is proposed. Similarly, a framework for reformulating Mixed-Integer Non-Linear Programming (MINLP) problems to a convex relaxed form is discussed in [14]. As common ideas, trying to avoid both bi-linear and non-linear terms into the problem reformulation is linked to the reduction of the number of decision variables and simplifying the nature of the proposed constraints from the definition stage of the optimisation problem.

Thus, the main contribution of this work is to provide three different ways of representing discrete feasible sets for control applications based on optimisation as well as to present a comparison of such approaches concerning the required computational burden for their implementation. In this regard, mathematical tools and representations such as rounding error and polyhedrons [15], [16] have been employed to constrain the discrete sets and to reduce the number of binary variables required when the optimisation problem is reformulated as a compact 0-1 MILP problem. The main idea is to reformulate the optimisation problem effectively and efficiently to simplify the input to optimisation routines such that the controllers can be suitable for their implementation in real time without the use of precomputed control laws. Other strategies have been excluded from this proposed comparative assessment due to their computational burden, a fact that does not allow their use in real time according to the field of application considered in this paper.

Then, to test the performance of the proposed approaches, an optimisation-based controller proposed in [17] to min- 
imise the energy consumption of a manufacturing process line will be considered. In this case, the optimal activation instants of binary and discrete actuators should be determined considering the operating constraints on both the process line and the system actuators. Thus, the optimisation problem is reformulated as an MILP problem using the proposed approaches, and the closed-loop performance for the controller is compared in terms of the control objective and the computational burden required to find an optimal solution.

The rest of the paper is organised as follows. The energy efficiency control strategy for manufacturing systems and the problem statement are introduced in Section II. Then, in Section III, the proposed approaches to model the discrete sets are presented. Next, the case study and the related decision models are introduced in Section IV. Later, the simulations results and the comparative assessment for the proposed approaches are presented and discussed in Section V. Finally, conclusions from the obtained results are presented in Section VI.

\section{ENERGY EFFICIENCY OF MANUFACTURING SYSTEMS}

\section{A. Energy Efficiency Control Strategy}

A process line is a complex system including several machine tools and peripheral devices that work sequentially and logically, as shown in Figure 1. Machines refer to a set of devices that are directly related to the machining processes (e.g., milling, cutting, turning), while the peripheral devices are those elements that provide the resources required to machines for their proper operation. Thus, there exist several functional relationships between machines and peripheral devices that determine the productivity of the process line.

Machine tools are characterised by periodic behaviour according to the time required to process a piece, which is denoted by $T_{M_{i}}$. Besides, due to the different operations performed by machine devices, there are high and low energy consumption stages along $T_{M_{i}}$. Then, since peripheral devices supply resources to machines in the process line, such devices might or might not show a periodic behaviour, which may match with $T_{M_{i}}$. Thus, to improve the energy efficiency of manufacturing system, a predictive-like controller to manage the activation instants of the peripheral devices in a process line without affecting its productivity has been proposed in [17]. In this case, the activation/deactivation of the machine devices along $T_{M_{i}}$, i.e., the machining sequence of the $i$-th machine $\left(\Lambda_{\mathbf{M}_{\mathbf{i}}}\right)$, is considered as fixed and periodic over time. Thus, $T_{M_{i}}$ are kept the same and machines can handle the same number of pieces as when the control strategy is not implemented. The energy consumption for $\boldsymbol{\Lambda}_{\mathbf{M}_{\mathbf{i}}}$, i.e., $\beta_{\mathbf{M}_{\mathbf{i}}}=\sum_{k=1}^{T_{M_{i}}} S_{M_{i}}(k)$, will also be constant over any interval $T_{M_{i}}$ and periodic over time, being $S_{M_{i}}$ the instantaneous power consumption of the machine.

The control strategy proposed in [17] focuses on the management of peripheral devices to minimise the total energy consumption and guarantee the supply of the resources required by the machines. Then, considering a fixed number of both machines and peripheral devices in a process line, their activation sequences can be defined as

$$
\begin{aligned}
\boldsymbol{\Lambda}_{\mathbf{M}_{\mathbf{i}}}(k) & =\left\{u_{M_{i, 1}}(k), u_{M_{i, 2}}(k), \ldots, u_{M_{i, v}}(k)\right\}, \\
\boldsymbol{\Lambda}_{\mathbf{P}}(k) & =\left\{u_{1}(k), u_{2}(k), \ldots, u_{m}(k)\right\},
\end{aligned}
$$

being $k \in \mathbb{Z}_{\geq 0}$ the discrete-time index, and $u_{M_{i, l}}(k)$ and $u_{j}(k)$ the activation signals of machining devices of the $i$-th machine and the peripheral devices in the process line. Then, considering a prediction horizon $H_{p}$, the sequence ${ }^{1}$ for $\boldsymbol{\Lambda}_{\mathbf{P}}$ along $H_{p}$ is defined as

$$
\boldsymbol{\Gamma}(k) \triangleq\left\{\boldsymbol{\Lambda}_{\mathbf{P}}(k \mid k), \ldots, \boldsymbol{\Lambda}_{\mathbf{P}}\left(k+H_{p}-1 \mid k\right)\right\},
$$

and an MPC controller is designed based on the following open-loop optimisation problem:

$$
\min _{\boldsymbol{\Gamma}(\mathbf{k})} \sum_{k=1}^{H_{p}} S(k) \Delta k
$$

subject to

$$
\begin{aligned}
\xi(k+r+1 \mid k)= & f\left(\xi(k+r \mid k), \boldsymbol{\Lambda}_{\mathbf{M}_{\mathbf{i}}}(k+r \mid k), \ldots\right. \\
& \left.u_{j}(k+r \mid k)\right), \\
S(k+r \mid k)= & g(\xi(k+r \mid k)), \\
Q_{j}(k+r+1 \mid k)= & q_{j}\left(Q_{j}(k+r \mid k), u_{j}(k+r \mid k), \ldots\right. \\
& \left.\boldsymbol{\Lambda}_{\mathbf{M}_{\mathbf{i}}}(k+r \mid k)\right), \\
u_{j}(k+r \mid k) \in & \boldsymbol{\Omega}_{j}=\left\{s_{j_{1}}, s_{j_{2}}, \cdots, s_{j_{n}}\right\}, \\
Q_{h}(k+r+1 \mid k) \in & {\left[\bar{Q}_{h} \underline{Q}_{h}\right], }
\end{aligned}
$$

for $r \in\left\{0,1,2, . ., H_{p}-1\right\}$, being $S \in \mathbb{R}$ the instantaneous power consumption of the whole process line given by

$$
S(k)=\left(\sum_{i=1}^{b} S_{M_{i}}(k)\right)+\left(\sum_{j=1}^{m} S_{j}(k)\right),
$$

with $S_{M_{i}} \in \mathbb{R}$ and $S_{j} \in \mathbb{R}$ the power consumption of the machines and peripheral devices, respectively. Besides, in (3), $\xi \in \mathbb{R}^{n_{1}}$ is the state vector of the energy consumption model of the process line, while $f: \mathbb{R}^{n_{1}} \times \boldsymbol{\Omega}_{j} \mapsto \mathbb{R}^{n_{1}}$ and $h: \mathbb{R}^{n_{1}} \mapsto \mathbb{R}_{\geq 0}$ are the maps for such a model. Moreover, $Q_{j}$ corresponds to states of the process dynamics related to the operation of peripheral devices with $q_{j}: \boldsymbol{\Omega}_{j} \times \mathbb{R} \mapsto \mathbb{R}_{\geq 0}$ the maps that consider the operational relationships between machines and peripheral devices. According to Figure 1, the $q$-relations in (3) correspond to the process dynamics for the supply systems of compressed air and coolant.

\section{B. Problem Statement}

It is worth noting that $(3 \mathrm{e})$ and (3f) refer to the constraints for the domain of decision variables and process variables $Q_{j}$, respectively. In this case, $\boldsymbol{\Omega}_{j} \subset \mathbb{R}$ is the discrete and countable finite set of cardinality $n_{j}$ describing the feasible domain of $u_{j}$, with $s_{j_{n}}$ the values (or symbols) that form such discrete (or alphabet) set. In the most simple case, $u_{j}$ will be constrained to a binary set, which represents the on/off status of a device with nominal power consumption.

\footnotetext{
${ }^{1}$ Here, $z(k+r \mid k)$ denotes the prediction of the variable $z$ at time instant $k+r$ performed at $k$. The index $r$ will cover the finite prediction window of length $H_{p}$.
} 

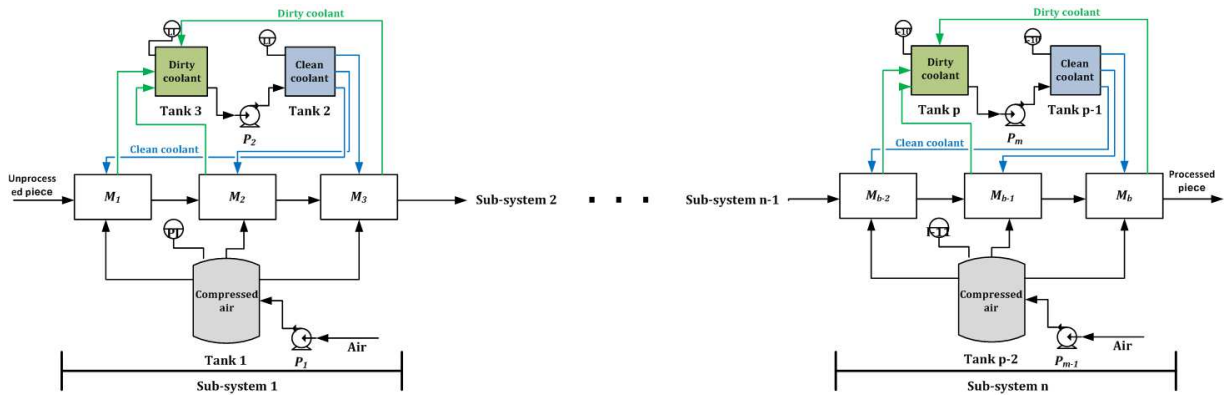

Fig. 1: A b-stage serial process line with its corresponding peripheral devices.

However, in a real plant, peripheral devices are of different nature and usually constrained to different discrete sets based on their design and the configuration of the process line.

Taking into account the admissible domain for the decision variables in (3), the problem addressed in this paper is to determine suitable ways of modelling these domains, fact that contributes to reducing the computational burden required to solve (3). This last fact is important for the real-time implementation of closed-loop control strategies based on optimisation, in which the optimisation problem should be solved fast enough at every instant $k$. Therefore, to get suitable representations of discrete sets such as $\boldsymbol{\Omega}_{j}$, the nature of the decision variables and corresponding mathematical expressions to represent the admissible values should be determined and added to the optimisation problem.

\section{Modelling of Discrete Sets}

Due to the nature of the decision variables in (3), three different ways to represent the discrete sets are presented below to formulate the optimisation problem as a compact 0-1 MILP problem. Although there are some optimisation routines that model directly such sets, the idea behind the proposed approaches is to further simplify the problem before to use the optimisation solver.

Approach 1: Rounding-error based strategy: In this case, to model $\boldsymbol{\Omega}_{j}$ the following statement is assumed:

Assumption 1: A discrete set $\boldsymbol{\Omega}_{j}=\left\{s_{j_{1}}, s_{j_{2}}, \cdots, s_{j_{n}}\right\}$ with $s_{j_{n}} \in \mathbb{R}$ is an ordered set in which all its elements have a regular spacing $\rho$.

Under this assumption, to model the discrete feasible set $\boldsymbol{\Omega}_{j}$, one decision variable, i.e., $\tilde{u}_{j} \in[\underline{\varepsilon}, \bar{\varepsilon}]$ with $\bar{\varepsilon}=$ $\max \left(\boldsymbol{\Omega}_{\mathrm{j}}\right)$ and $\underline{\varepsilon}=\min \left(\boldsymbol{\Omega}_{\mathrm{j}}\right)$ is required. Besides, to ensure that only the admissible values of $\boldsymbol{\Omega}_{j}$ in $[\underline{\varepsilon}, \bar{\varepsilon}]$ could be selected, the rounding error should be added as a constraint into the optimisation problem in (3) as follows:

$$
\left|\rho\left[\left(\frac{\tilde{u}_{j}}{\rho}\right)\right]-\tilde{u}_{j}\right| \leq 0,
$$

with $[\cdot]$ the round operator and $\rho$ the regular spacing among the elements of $\boldsymbol{\Omega}$. Based on the previous inequality, only the values in $[\underline{\varepsilon}, \bar{\varepsilon}]$ that satisfy $(x \bmod \rho) \equiv 0$ can be chosen.

Remark 1: If zero belongs to $\boldsymbol{\Omega}_{j}$ and its distance to the next element in $\boldsymbol{\Omega}_{j}$ does not satisfy the spacing regularity of the rest of the elements, a new variable and a constraint should be introduced, e.g., when zero represents the off status and the rest of the elements refer to different activation levels. Thus, zero is considered separately by its nilpotent properties within $\boldsymbol{\Omega}_{j}$, and an extra binary variable $\phi_{j}$ and a new constraint are added as follows:

$$
\underline{\varepsilon} \phi_{j} \leq \tilde{u}_{j} \leq \bar{\varepsilon} \phi_{j},
$$

with $\phi_{j} \in\{0,1\}$ and $\tilde{u}_{j} \in[\underline{\varepsilon}, \bar{\varepsilon}]$. Besides, this approach could be extended to the case in which $\rho$ for the elements of $\boldsymbol{\Omega}_{j}$ is not regular. The original set is split into subsets with regular spacing, which are associated with one binary variable, rounding error equation, and extra constraints on binary variables to ensure the selection of only one set.

Approach 2: Direct binary approach encoding: Given a set $\boldsymbol{\Omega}_{j}=\left\{s_{j_{1}}, s_{j_{2}}, \cdots, s_{j_{n}}\right\}$ with $s_{j_{n}} \in \mathbb{R}$, one can associate $n$ decision variables, i.e., $\gamma_{1}, \gamma_{2}, \cdots, \gamma_{n} \in\{0,1\}$, and reinforce the alternative of decision with the constraint

$$
\gamma_{1}+\gamma_{2}+\cdots+\gamma_{p}=1 .
$$

Indeed, to guarantee the selection of only one of the values in $\boldsymbol{\Omega}_{j}$, the decision variable $u_{j}$ is computed according to

$$
u_{j}=s_{j_{1}} \gamma_{1}+s_{j_{2}} \gamma_{2}+\cdots+s_{j_{n}} \gamma_{n} .
$$

Remark 2: It should be noted that if zero belongs to $\boldsymbol{\Omega}_{j}$, $n-1$ binary variables are needed, and the constraint in (7) should be relaxed as $\gamma_{1}+\gamma_{2}+\cdots+\gamma_{n-1} \leq 1$.

Approach 3: Geometrical representation of the feasible domain: In this case, the discrete set will be modelled by means of a polyhedron given as the intersection of inequalities and equalities (referred to as H-representation). To get a polyhedral representation of a discrete set, auxiliary variables are defined to fit the vertices of the polyhedron. For instance, considering a discrete set $\boldsymbol{\Omega}_{j}=\left\{s_{j_{1}}, s_{j_{2}}, s_{j_{3}}, s_{j_{4}}\right\}$, two binary variables and one real variable, i.e., $\delta_{1}, \delta_{2} \in$ $\{0,1\}$, and $u_{j} \in \mathbb{R}$, are required. Next, the discrete values of $\Omega_{j}$ will be associated to the combinations of variables $\delta_{1}$ and $\delta_{2}$, which define the vertices set $V$ of the polyhedron as

$$
V=\left\{\left[0,0, s_{j_{1}}\right] ;\left[0,1, s_{j_{2}}\right] ;\left[1,0, s_{j_{3}}\right] ;\left[1,1, s_{j_{4}}\right]\right\} .
$$

Then, using suitable tools, such as the MPT Toolbox [18], the H-representation of the polyhedron $P_{v}=$ $\left\{v \mid A v \leq b, A_{e} v=b_{e}\right\}$, which corresponds to the convex hull of $V$, can be determined. These expressions should be added as constraints in the optimisation problem such that $\left[\delta_{1}, \delta_{2}, u_{j}\right]^{T} \in P_{v}$. Based on this formulation, for any combination of the binary variables only the values in 
$\boldsymbol{\Omega}_{j}$ satisfy the inequality and equality matrices added as constraints.

\section{Benchmark System}

According to Figure 1, the process line is formed by $n$ subsystems, which consists of three machines and two shared peripheral devices. Thus, the process description presented below for the one sub-system is straightforwardly extended to the other sub-systems without lost of generality. It is assumed that all machines in each sub-system have the same period, i.e., $T_{M_{i}}=28 s \forall i=1,2,3$, and that the peripheral devices $P_{1}$ and $P_{2}$ are shared among these machines. In this case, $P_{1}$ is associated to a supply system of compressed air with nominal energy consumption, i.e., $u_{1} \in \boldsymbol{\Omega}_{1} \triangleq\{0,1\}$. Thus, the $q$-relations in (3d) related to $P_{G_{1}}$ refer to the dynamics for the total change of mass $M_{T_{1}}$ and pressure $P_{T_{1}}$ inside a storage $\operatorname{tank} T_{1}$, which are expressed as follows:

$$
\begin{aligned}
& M_{T_{1}}(k+r+1 \mid k)=M_{T_{1}}(k+r \mid k)+\tau_{s} \sigma(k+r \mid k), \\
& \sigma(k+r \mid k)=m_{\text {in }} u_{G_{1}}(k+r \mid k)-\sum_{i=1}^{b} m_{\text {out }_{M_{i}}}(k+r \mid k), \\
& P_{T_{1}}(k+r \mid k)=\frac{M_{T_{1}}(k+r \mid k) R T}{V_{T_{1}} W_{\text {air }}}
\end{aligned}
$$

with $\tau_{s}$ the sampling time, $m_{\text {out }_{M_{i}}}$ the air consumption from $M_{i}, m_{i n}$ the air flow pumped by $P_{G_{1}}$ towards the tank $T_{1}$, and, $R, T, V_{T_{1}}$, and $W_{\text {air }}$ the gas constant, air temperature, volume of $T_{1}$, and the molecular weight of the air, respectively. Besides, $P_{T_{1}}$ must belong to $\left[\underline{P}_{T_{1}}, \bar{P}_{T_{1}}\right]$.

Besides, $P_{2}$ is related to a coolant supply system for the cutting operations at each machine, and its activation can be modulated at different levels, i.e., $u_{2} \in \Omega_{2} \triangleq$ $\{0,100,120,140\}$. According to Figure 1 , a flow of coolant $m_{c}$ is pumped by $P_{2}$ from $T_{3}$ towards $T_{2}$, passing through a filter in which the gross particles are separated. The coolant flows required by the machines are pumped from $T_{2}$. The level dynamics in both tanks are given by

$$
\begin{aligned}
& L_{2}(k+r+\mid k)=L_{2}(k+r \mid k)+\tau_{s} \gamma(k+r \mid k)\left(\frac{1}{\rho_{c} A_{T_{2}}}\right), \\
& \gamma(k+r \mid k)=m_{c}(k+r \mid k)-\sum_{i=1}^{b} m_{\text {out }, c_{M_{i}}}(k+r \mid k), \\
& L_{3}(k+r+1 \mid k)=L_{3}(k+r \mid k)+\tau_{s} \theta(k+r \mid k)\left(\frac{1}{\rho_{c} A_{T_{3}}}\right) \\
& \theta(k+r \mid k)=\sum_{i=1}^{b} m_{\text {in },_{M_{i}}}(k+r \mid k)-m_{c}(k+r \mid k), \\
& P_{\text {out }}(k+r \mid k)=P_{\text {in }}(k+r \mid k)+\rho_{c} h_{f_{1 \rightarrow 2}}(k+r) \\
& \quad-\eta\left(\frac{W(k+r \mid k)}{m_{c}(k+r \mid k)}\right),
\end{aligned}
$$

with $m_{\text {out }, c_{M_{i}}}$ the coolant flow required by the $i$-th machine, and $m_{i n, c_{M_{i}}}$ the flow of the dirty coolant recovered. Besides, $P_{i n}$ and $P_{\text {out }}$ correspond to the input and output pressure in the pipe system from $T_{3}$ towards $T_{2}$, while, $\rho_{c}, \eta, W$ and $h_{f_{1 \rightarrow 2}}$ are the coolant density, the pump efficiency, the work supply to $P_{G_{2}}$, and the energy losses by friction, respectively.

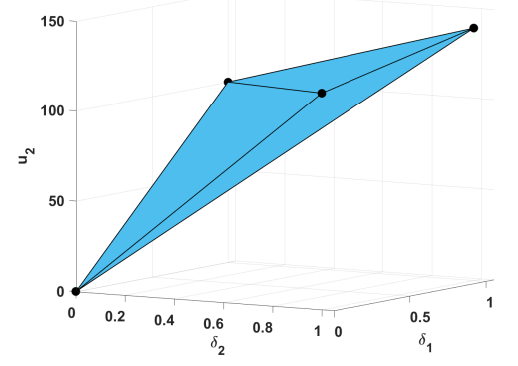

Fig. 2: Polyhedron for the discrete set $\Omega_{2}$.

\section{A. Discrete domain for the decision variables}

Given the nature of the discrete sets $\boldsymbol{\Omega}_{1}$ and $\boldsymbol{\Omega}_{2}$, the decision variables and constraints required by each one of the approaches proposed in Section III are presented below.

Approach 1: If zero is removed from the set $\Omega_{2}$ and an extra variable is added for the switching off of $P_{2}$, i.e. $u_{2}=$ 0 , it is possible to fix $\rho=20$ for the elements of $\Omega_{2}$. Thus, three decision variables are required to model $\boldsymbol{\Omega}_{1}$ and $\boldsymbol{\Omega}_{2}$, i.e., $u_{1}(k), \phi_{2}(k) \in\{0,1\}$, and $\tilde{u}_{2}(k) \in[100,140]$, with $u_{1}$ related to $P_{1}$ and, $\phi_{2}$ and $\tilde{u}_{2}$ associated to $\boldsymbol{\Omega}_{2}$. Then, considering $\bar{\varepsilon}=100$ and $\underline{\varepsilon}=140$, constraints in (5) and (6) should be adapted and added to (3).

Approach 2: In this "full" binary encoding, four binary variables are required to represent the domain of decision variables, i.e., $u_{1}(k), \gamma_{1}(k), \gamma_{2}(k), \gamma_{3}(k) \in\{0,1\}$, with $u_{1}$ related to the device $P_{1}$, and according to Remark 2 , three binary variables $\gamma_{1}, \gamma_{2}$ and $\gamma_{3}$ are required to model the discrete set $\boldsymbol{\Omega}_{2}$ together with the following constraint:

$$
\gamma_{1}(k)+\gamma_{2}(k)+\gamma_{3}(k) \leq 1 .
$$

Finally, $u_{2}$ is given by

$$
u_{2}(k)=100 \gamma_{1}(k)+120 \gamma_{2}(k)+140 \gamma_{3}(k) .
$$

Approach 3: In this case, one binary variable for the activation/deactivation of $P_{1}$ is required, i.e., $u_{1}(k) \in\{0,1\}$, while two binary variables, i.e., $\delta_{1}(k), \delta_{2}(k) \in\{0,1\}$, and one real variable, i.e., $\tilde{u}_{2}(k) \in \mathbb{R}$, are needed to model $\boldsymbol{\Omega}_{2}$. Then, each combination of $\delta_{1}$ and $\delta_{2}$ will be related to one element in the set $\boldsymbol{\Omega}_{2}$, and a polyhedron with vertices

$$
V_{P_{G_{2}}}=\{[0,0,0][0,1,100][1,0,120][1,1,140]\}
$$

is defined and shown in Figure 2. Next, using the MPT toolbox, the H-representation of the polyhedron was obtained and, the following inequality matrix is added to (3):

$$
\left[\begin{array}{cccc}
-0.768 & -0.640 & 0.006 & 0 \\
0.986 & 0.164 & -0.008 & 0 \\
0.371 & 0.928 & -0.009 & 0 \\
-0.436 & -0.218 & 0.011 & 0.873
\end{array}\right]\left[\begin{array}{c}
\delta_{1} \\
\delta_{2} \\
\tilde{u}_{2} \\
-1
\end{array}\right] \leq\left[\begin{array}{l}
0 \\
0 \\
0 \\
0
\end{array}\right]
$$

\section{B. Energy consumption models}

Data-driven models were used to identify the power consumption models of machines and peripheral devices. In this case, Subspace Identification (SI) methods were used 
since they allow identifying the matrices of a state-space realisation of linear time-invariant systems based on inputoutput data [19]. According to [17], different sequences of $\boldsymbol{\Lambda}_{\mathbf{M}_{\mathbf{i}}}$ along $T_{M_{i}}$ were designed and tested in a test bench that emulates the energy consumption of machine tools to collect the data of $S_{M_{i}}$. Besides, different sequences of $\boldsymbol{\Lambda}_{\mathbf{P}}$ were tested to obtain a rich range of the outputs $S_{P_{j}}$. From the obtained input-output data sets, energy consumption models were identified by using the n $4 \mathrm{sid}$ routine of the System Identification Toolbox ${ }^{\mathrm{TM}}$ provided by Matlabß). Thus, different values of $N$ were tested to identify the matrices that allow the highest fitting degree between the real and modelled outputs. The energy consumption models are detailed in [17].

\section{Simulation Results}

The control strategy was tested using each one of the proposed approaches to compare their performance and computational burden. All simulations were performed using an Intel Core i7-55000U $2.4 \mathrm{GHz}$ processor with 8G RAM and considering a sampling time equal $\tau_{s}=0.1 \mathrm{~s}$. The simulation results were obtained in Matlab by using the software IBM ILOG CPLEX Optimisation Studio integrated to YALMIP toolbox [20]. Since the controller was designed to make decisions every second along $H_{p}=28 s$, a faster internal loop (blocking) for both the process and energy consumption models are implemented [21].

The resulting energy consumption profile and the optimal activation/deactivation sequence of peripheral devices are presented in Figures $3 \mathrm{a}$ and $3 \mathrm{~b}$ for the case in which $n=$ 1. Besides, in Figure $3 \mathrm{c}$ the process dynamics related to both $P_{G_{1}}$ and $P_{G_{2}}$ are shown. From these results, some differences can be observed for the peripheral device with a discrete domain, i.e., $P_{G_{2}}$, for which even when the device was turned on at the same time instant, the activation value was different according to the approach tested. Thus, although the stopping criteria of optimisation routine were the same for all approaches, these differences could be related to the solver ability to test all possible combinations for each approach before to reach the stopping criteria. This is due to the fact that, for each modelling approach, the nature and the total number of decision variables are different and, therefore, the number of the possible combinations could differ although the feasible domain is similar.

Next, to compare the computational burden when more decision variables are considered, a process line adding subsystems as shown in Figure 1 is studied. It should be noted that for each one of the sub-systems added, the operating conditions, machine cycles, energy consumption models, and dynamics of peripheral devices are assumed to be the same that for the first subsystem with three machines and two peripheral devices. In Table I, a comparison of the proposed approaches concerning both the computational burden and the control objective is presented when several sub-systems are considered as the process line. In this regard, the decision variables (DV) along $H_{p}$, their classification, the number of equality and inequality constraints, the CPU time and the value of the cost function are presented in Table I.
In Table I, the auxiliary variables (AV) refers to the variables added to model the non-linear operators involved in the rounding error expression. Thus, the total number of decision variables considered by each approach corresponds to the set of both the decision and auxiliary variables. Besides, for Approach 1 all the auxiliary variables added to the decision model were defined as integer variables and by the optimisation routine internally. Finally, the total number of continuous variables (CV) for Approaches 1 and 3 are summarised in the seventh column. From these results, it should be noted that the equality constraints are the same for all approaches since they correspond to the initial conditions for both the process model and the machining sequences.

Based on the results in Table I, it is possible to observe that even when the optimal activation sequences of peripheral devices obtained from each approach are different, the total energy consumption is similar for each one of the approaches tested. However, higher differences can be observed in the CPU time spent to solve the optimisation problem at each iteration. Thus, although the Approach 1 has a higher number of total decision variables, the solution is obtained faster than for the rest of cases. This behavior is related to the fact that Approach 1 has a lower number of binary variables, and therefore, fewer combinations should be tested and the completion conditions are achieved faster. Therefore, when the branch-and-bound algorithms are employed, the computational burden to evaluate all (or most of) the combinations is increased as the binary variables increase. In this regard, by implementing the proposed Approach 1 with fewer number of binary variables, the computational burden can be reduced even when auxiliary variables are required. However, although both Approaches 2 and 3 require more binary variables to model $\Omega_{2}$ than Approach 1 , the latter is more restrictive than the other approaches. Thus, when more complex discrete sets with no regular spacing are analysed, Approach 1 could require more binary variables than Approaches 2 and 3, in addition to the auxiliary variables due to both the rounding operator and absolute value.

\section{Conclusions}

In this paper, three different approaches to reformulate optimisation problems involving discrete and continuous variables into a mixed-integer MILP problem have been presented. Based on the proposed formulations, ordered and non-ordered discrete sets could be modelled by using rounding error, binary variables or polyhedral approximations. Besides, the necessary constraints to guarantee the selection of the discrete admissible values according to each approach as well as the conditions for their implementation were presented and discussed. Based on the obtained results, it is possible to conclude that the total amount of binary variables is a crucial factor regarding the computational burden even when the total number of decision variables is lower. However, despite the computational cost, the binary variables lead to optimisation problems that involve non-ordered and nonregularly distributed symbol sets can be solved in an efficient manner and with a manageable computational burden. 


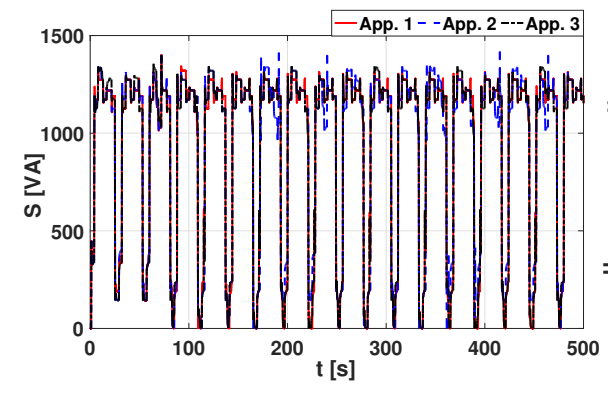

(a) Instantaneous power consumption profile.

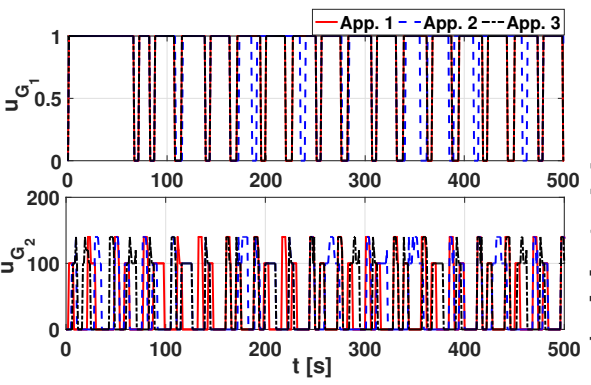

(b) Optimal input sequences.

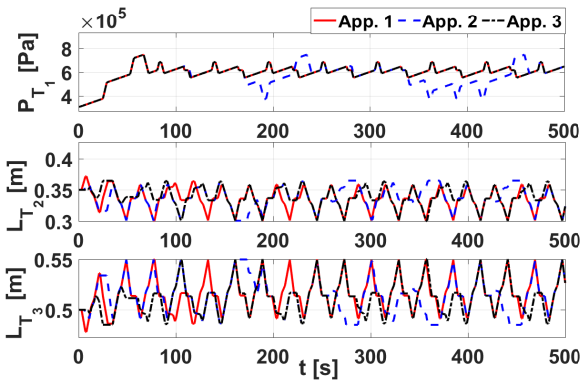

(c) Dynamics of peripheral devices.

Fig. 3: Simulation results of the control strategy using the proposed approaches.

TABLE I: Comparison of the proposed approaches regarding their performance and computational burden.

\begin{tabular}{|c|c|c|c|c|c|c|c|c|c|c|c|}
\hline App. & $\begin{array}{c}\text { Subsystem } \\
\text { n }\end{array}$ & $\begin{array}{c}\text { No. } \\
\text { Devices }\end{array}$ & $\begin{array}{c}\text { No. DV } \\
\text { along } H_{p}\end{array}$ & $\begin{array}{c}\text { No. } A V \\
\text { along } H_{p}\end{array}$ & $\begin{array}{l}\text { No. BV } \\
\text { along } H_{p}\end{array}$ & $\begin{array}{c}\text { No. CV } \\
\text { along } H_{p}\end{array}$ & $\begin{array}{c}\text { No. inequality } \\
\text { constraints }\end{array}$ & $\begin{array}{c}\text { No. equality } \\
\text { constraints }\end{array}$ & $\begin{array}{c}\max \text { CPU } \\
\text { time (s) }\end{array}$ & $\begin{array}{c}\text { CPU } \\
\text { time (s) }\end{array}$ & $\mathbf{S}[\mathbf{V A}]$ \\
\hline 1 & 1 & 2 & 87 & 58 & 58 & 29 & 1033 & 1977 & 0.157 & 43.278 & 1057389.179 \\
\hline 2 & 1 & 2 & 116 & 0 & 116 & 0 & 975 & 1977 & 0.563 & 119.064 & 1057631.542 \\
\hline 3 & 1 & 2 & 116 & 0 & 87 & 29 & 946 & 1977 & 0.390 & 84.978 & 1057389.179 \\
\hline 1 & 2 & 4 & 174 & 116 & 116 & 58 & 2066 & 3954 & 0.875 & 241.582 & 2114885.914 \\
\hline 2 & 2 & 4 & 232 & 0 & 232 & 0 & 1950 & 3954 & 1.172 & 424.487 & 2114843.857 \\
\hline 3 & 2 & 4 & 232 & 0 & 174 & 58 & 1892 & 3954 & 1.609 & 661.855 & 2114506.408 \\
\hline 1 & 3 & 6 & 261 & 174 & 174 & 87 & 3099 & 5931 & 2.375 & 581.211 & 3172410.754 \\
\hline 2 & 3 & 6 & 348 & 0 & 348 & 0 & 2925 & 5931 & 3.078 & 1004.515 & 3171807.409 \\
\hline 3 & 3 & 6 & 348 & 0 & 261 & 87 & 2838 & 5931 & 4.781 & 1019.072 & 3171530.959 \\
\hline 1 & 4 & 8 & 348 & 232 & 232 & 116 & 4132 & 7908 & 2.953 & 605.873 & 4229758.495 \\
\hline 2 & 4 & 8 & 464 & 0 & 464 & 0 & 3900 & 7908 & 2.891 & 1103.728 & 4229160.288 \\
\hline 3 & 4 & 8 & 464 & 0 & 348 & 116 & 3784 & 7908 & 1.983 & 1105.196 & 4229503.581 \\
\hline 1 & 5 & 10 & 453 & 290 & 290 & 145 & 5165 & 9885 & 2.844 & 603.630 & 5286723.512 \\
\hline 2 & 5 & 10 & 580 & 0 & 580 & 0 & 4875 & 9885 & 2.562 & 1274.541 & 5286230.569 \\
\hline 3 & 5 & 10 & 580 & 0 & 435 & 145 & 4730 & 9885 & 2.218 & 978.293 & 5286553.431 \\
\hline
\end{tabular}

\section{ACKNOWLEDGEMENT}

This work has been partially funded by the project IKERCON (ref. C10683) and and the FI-AGAUR scholarship of the Catalan government.

\section{REFERENCES}

[1] C. Ning and F. You, "Data-driven robust MILP model for scheduling of multipurpose batch processes under uncertainty," in 2016 IEEE 55th Conference on Decision and Control (CDC), Dec 2016, pp. 61806185.

[2] C. Lu, L. Gao, X. Li, Q. Pan, and Q. Wang, "Energy-efficient permutation flow shop scheduling problem using a hybrid multi-objective backtracking search algorithm," Journal of Cleaner Production, vol. 144, pp. 228-238, 2017.

[3] C. A. Hans, P. Braun, J. Raisch, L. Grüne, and C. Reincke-Collon, "Hierarchical distributed model predictive control of interconnected microgrids," IEEE Transactions on Sustainable Energy, vol. 10, no. 1, pp. 407-416, Jan 2019.

[4] G. C. Goodwin, D. Q. Mayne, T. Chen, C. Coates, G. Mirzaeva, and D. E. Quevedo, "Opportunities and challenges in the application of advanced control to power electronics and drives," in 2010 IEEE International Conference on Industrial Technology, March 2010, pp. $27-39$.

[5] J. L. Diaz C. and C. Ocampo-Martinez, "Energy efficiency in discretemanufacturing systems: Insights, trends, and control strategies," Journal of Manufacturing Systems, vol. 52, pp. 131 - 145, 2019.

[6] J. Rawlings and D. Mayne, Model Predictive Control: Theory and Design. Madison, WI (USA): Nob Hill Publishing, 2009.

[7] J. B. Rawlings and M. J. Risbeck, "Model predictive control with discrete actuators: Theory and application," Automatica, vol. 78, pp. 258-265, 2017.

[8] I. Prodan, F. Stoican, S. Olaru, and S.-I. Niculescu, "Enhancements on the hyperplanes arrangements in mixed-integer programming techniques," Journal of Optimization Theory and Applications, vol. 154, no. 2, pp. 549-572, 2012.
[9] S. Diamond, R. Takapoui, and S. Boyd, "A general system for heuristic minimization of convex functions over non-convex sets," Optimization Methods and Software, vol. 33, no. 1, pp. 165-193, 2018.

[10] S. Yildız and J. P. Vielma, "Incremental and encoding formulations for mixed integer programming," Operations Research Letters, vol. 41, no. 6, pp. 654-658, 2013.

[11] Q. An, S.-C. Fang, H.-L. Li, and T. Nie, "Enhanced linear reformulation for engineering optimization models with discrete and bounded continuous variables," Applied Mathematical Modelling, vol. 58, pp. $140-157,2018$.

[12] I. Prodan, F. Stoican, S. Olaru, and S.-I. Niculescu, Mixed-Integer Representations. Cham: Springer International Publishing, 2016, pp. $35-56$.

[13] H.-L. Li, Y.-H. Huang, and S.-C. Fang, "Linear reformulation of polynomial discrete programming for fast computation," INFORMS Journal on Computing, vol. 29, no. 1, pp. 108-122, 2016.

[14] A. Lundell and T. Westerlund, "Solving global optimization problems using reformulations and signomial transformations," Computers \& Chemical Engineering, vol. 116, pp. 122-134, 2018.

[15] A. Bemporad, K. Fukuda, and F. D. Torrisi, "Convexity recognition of the union of polyhedra," Computational Geometry, vol. 18, no. 3, pp. $141-154,2001$.

[16] M. Lubin, E. Yamangil, R. Bent, and J. P. Vielma, "Polyhedral approximation in mixed-integer convex optimization," Mathematical Programming, vol. 172, no. 1-2, pp. 139-168, Sept. 2017.

[17] J. L. Diaz C., M. Bermeo, J. Diaz-Rozo, and C. Ocampo-Martinez, "An optimization-based control strategy for energy efficiency of discrete manufacturing systems," ISA Transactions, vol. 93, pp. 399 - 409, 2019.

[18] M. Kvasnica, P. Grieder, and M. Baotić, "Multi-Parametric Toolbox (MPT)," 2004. [Online]. Available: http://control.ee.ethz.ch/ mpt/

[19] S. Qin, "An overview of subspace identification," Computers \& Chemical Engineering, vol. 30, no. 10, pp. 1502-1513, 2006, papers form Chemical Process Control VII.

[20] J. Löfberg, "Yalmip : A toolbox for modeling and optimization in Matlab," in In Proceedings of the CACSD Conference, Taipei, Taiwan, 2004

[21] J. Maciejowski, Predictive Control with Constraints. Great Britain: Prentice Hall, 2002. 